\title{
Design of double loop-locked system for brush-less DC motor based on DSP
}

\author{
Yunhong Zheng ${ }^{1,}$, Ziqiang $\mathrm{Hua}^{2}$ and $\mathrm{Li} \mathrm{Ma}^{3}$ \\ ${ }^{1}$ School of Electrical Engineering and Automation, Xiamen University of Technology, Xiamen 361024 , \\ China \\ ${ }^{2}$ School of Electrical Engineering and Automation, Xiamen University of Technology, Xiamen 361024, \\ China
}

\begin{abstract}
In order to improve the control effect of the brush-less DC motor, adjustable-speed system of double loop-locked based on TMS320f28335 chip is designed to realize the digital control of the brush-less DC motor. As for the hardware part, the TMS320f28335 chip is the core processor and the brush-less DC motor is drived by three-phase bridge circuit. As for the software part, PID algorithm is applied to realize the double loop-locked control of the motor's location and RMP. The conclusion is proved by the experiments that the adjustable-speed system can run steadily and its control algorithm is reasonable, which has a broad application prospect.
\end{abstract}

Keywords: TMS320F28335; the brush-less DC motor; PID algorithm; the double loop-locked control.

\section{Introduction}

Due to its simple structure, high efficiency, long life and low noise characteristics, brush-less DC motor has been widely used in the defense, aerospace, robotics, automotive electronics, precision machine tools and other fields. At present, the control of brush-less DC motor mainly adopts special-purpose integrated circuit controller. Application-specific integrated circuit controller has a simple structure, high cost performance, peripheral devices speed. The experiments shown that the adjustable-speed system could run steadily and the control algorithm was effective.

Less than separate controller, but in the applications it will be affected by the expand ability of function and it's not easy to upgrade the product function changes and operation aspects, and more toward design of their own system hardware and software aspects, FPGA, MCU control system are often used for the motor. Under the widely application of the DSP, it's high data processing ability and powerful peripheral processing speed make it gradually becoming the first choice in the field of motor control. This paper is a design for the brush-less DC motor speed control system in which the TMS320F29335 is the core chip.

a Corresponding author : 2010120601@xmut.edu.cn

(C) 2016. The authors - Published by Atlantis Press 


\section{Work principle}

The equation of motion of knowable by dc motor: acceleration is proportional to the torque of the motor, and the torque is proportional to the motor current. Therefore, to achieve high accuracy, high dynamic performance of motor control, you need to control the speed of the motor at the same time, current and position for testing and control. Figure 1 is a brush-less DC motor control system block diagram, the system has been set up in the speed regulator and current regulator to adjust speed and current respectively, both between the cascade connection, the speed regulator output as a current regulator input, then use the output of the current regulator to control PWM unit. As is shown in figure 1, the system control unit can be divided into two parts: the dotted box function of TMS320F28335 DSP minimum system, it includes DSP and external storage, others for feedback signal acquisition part. Current feedback signal is measured by the hall element, through A/D module F28335 into digital quantity, rotor position signal is used to generate the correct rotor commutator, photoelectric encoder detection and feedback back to the motor rotation direction and Angle into DSP system, form a closed loop control. System position is given by the upper machine. Three-phase ac input after rectifying, voltage regulator to provide dc power for invert circuit, invert circuit of trigger signal provided by the upper machine, the purpose of which is the output of adjustable duty ratio of PWM signal, by adjusting the width of the PWM signal to control the on and off time of power tube, so as to realize control of the brush-less motor.

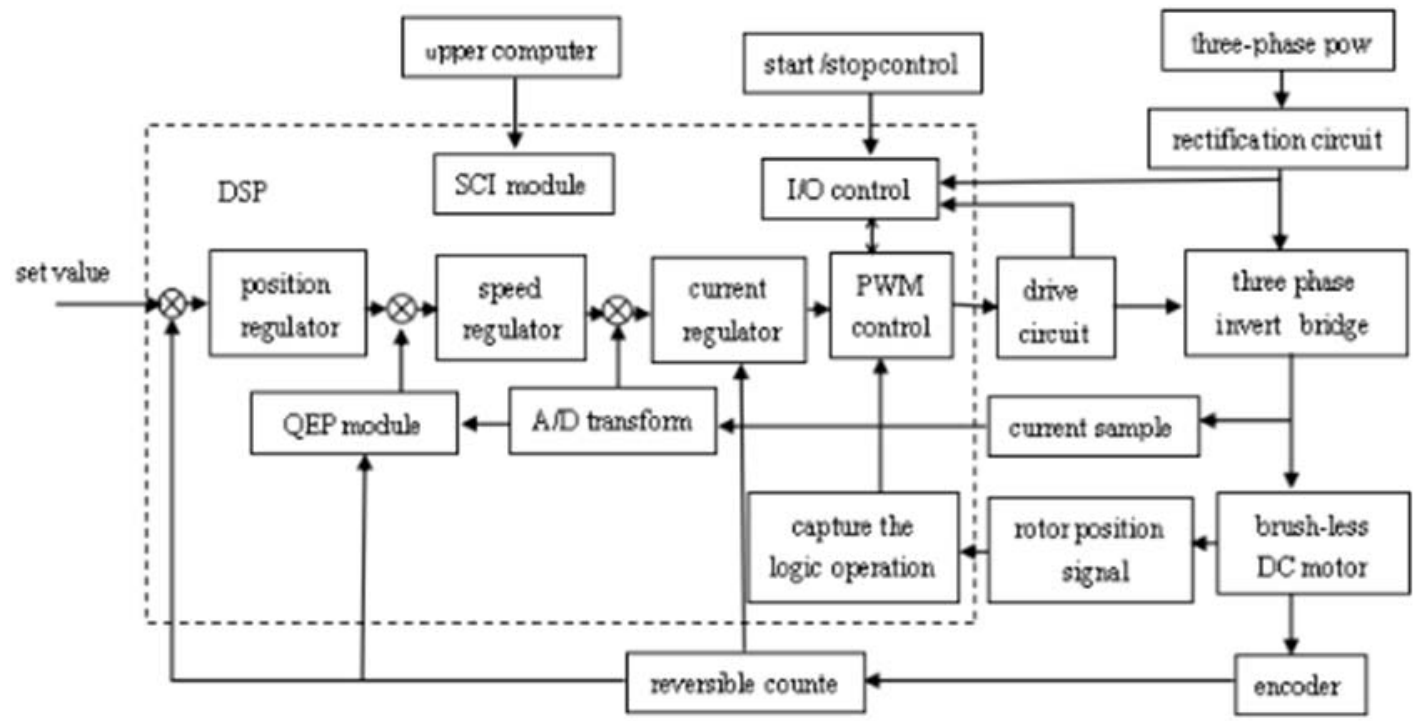

Figure 1. The system hardware block diagram

\section{Control process}

In the system control scheme to TMS320F28335 microcontroller as the core processor of control system, with power MOSFET tube invert, power device on the order of the V1 bridge arm, V3, V5, V2, under the bridge arm V4, the order of the V6, according to certain logical relationship open six power devices. According to the magnetic pole position sensor information, there are 6 kinds of state, one to one correspondence switch combination in bridge arm: V1V6, V6V3, V3V 2, V2V5 V5V4, V4V1, V1V6... The rotor each turned a pair of $\mathrm{N}-\mathrm{S}$ pole, V1 V6 power tube is fixed into six state conduction in turn. Each state, the only two phase winding electricity, in turn, change a state, the stator winding of the magnetic field generated along the axis of rotation Angle 60 degrees, so cycle, brush-less DC motor will produce continuous torque, to drag load for continuous rotation, which in turn inversion can be realized. 
The figure 2 is a typical double closed loop speed regulation system, including a speed regulation loop and a current adjusting ring. First of all, according to the detected the rotor position signal, the current rotating speed of the motor is calculated, and then compared with the speed reference speed deviation, after a PID controller to get the corresponding current reference value. The current reference value compared with the actual motor current feedback value, deviation of value after the PID will generate PWM signal to the power of the motor main circuit. By controlling the opening sequence and time of power tube, it can change the size of the motor stator winding current and winding conduction sequence, so as to realize control of the brush-less DC motor speed.

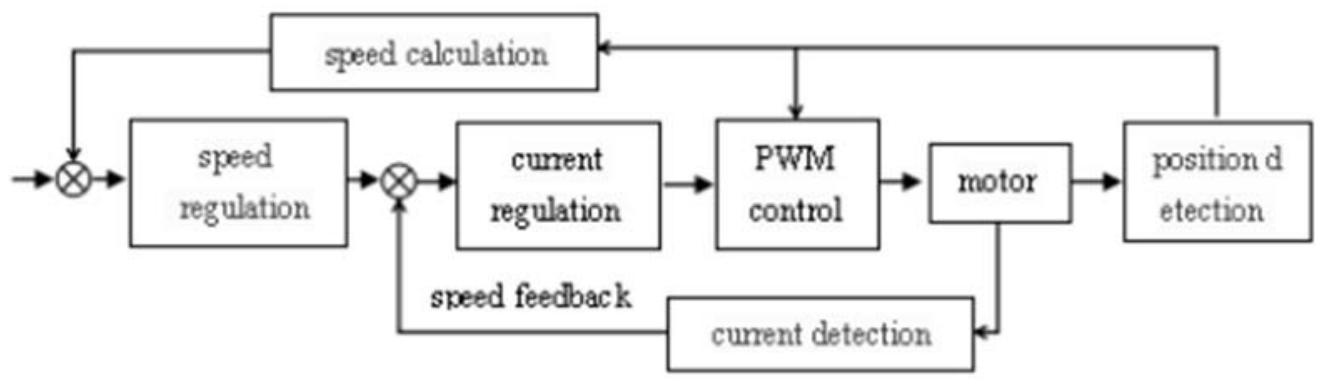

Figure 2. Dual-loop control system of FIG

\section{The realization of the system modules}

\subsection{Current test and calculation}

Current detection is to use high precision of measuring resistance, will detect resistors in series to dc bus. the choice of Resistance to consider when the over-current occurs can output the maximum voltage.

Each period of current sampling again. In the design of the PWM period is 50 us, that is the frequency of current sampling is $20 \mathrm{kHz}$. Switch tube adopts PWM control, the carrier is the triangular wave. At the instant of the PWM period "on", is not stable and not easy sampling current rise. So current sampling time should be in "open" in the middle of the PWM period, as shown in figure 3 the arrow is the current sampling time. Current sampling by DSP timer adopts the continuous increase or decrease in counting method matching event start the ADC conversion period.

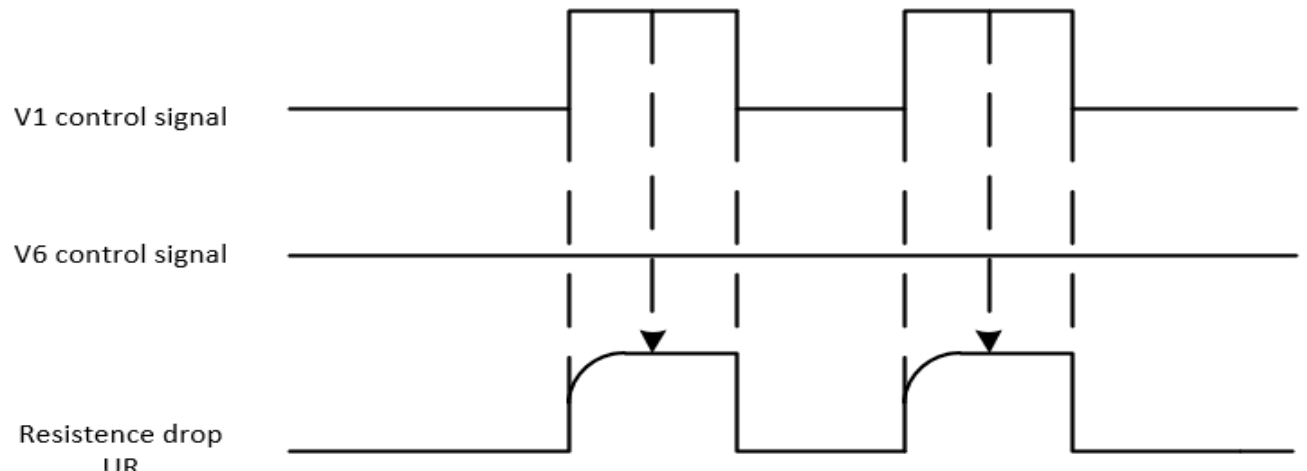

UR

Figure 3. A schematic view of the current sampling time 


\subsection{Position detection and calculation of speed}

Brush-less DC motor need to continually for three-phase brush-less DC motor reversing, mastering proper commutation time can reduce the torque fluctuation, so position detection is very important. At the same time through the position detection signal data, we can calculate the present speed of the motor. Position signal is obtained by three hall sensors. Each hall sensors will generates 180 degrees pulse width of output signal, three hall sensor output signal with 120 degrees phase difference. By setting DSP to double edge triggered capture interrupt function, gain the six moments. CAP1 CAP3 of the DSP are set as I/O ports, and detect the state of ports, the state of each level of hall sensor is known and the position of the rotor current is obtained.

Using the position signal to calculate the present speed of the motor. each of the motor mechanical has six times commutation, that is, the rotor turns 180 angle of each mechanical commutation, there is a measured by DSP timer twice commutation interval, twice during the commutation interval can be calculated the average angular velocity.

\subsection{Other module design}

There are two ways for the Motor speed setting in the system, one is with the method of potential scoring pressure, due to the voltage of A/D module DSP28335 sampling range is $0 \sim 3.3 \mathrm{~V}$, so the potentiometer is supplied by 3.3V. Another method is to use DSP28335 built-in SCI module communicates with the PC, using RS232 for communication. RS232 bus USES the differential transmission, strong anti-jamming capability, long transmission distance. The Settings of the current sampling also USES DSP28335 A/D module, potential scoring pressure.

\section{The software design}

The design of the software system mainly includes two parts: the speed calculation procedures and the double loop-locked control program of brush-less DC motor. We can achieve the calculation of speed parameter and the reversing operation by the speed calculating program. The rotor will trigger the capture interrupt every 60 degrees mechanical angle. In the capture interrupt subroutine, we should compute the parameter of speed first, then set the CAP pins to the I/O status. Now the position statue of the motor rotor would be read out when CAP pins were int the I/O status. Now we can achieve the reversing operation by the position statue of the motor rotor. Finally, we should restore the capture function of the CAP function. The double loop-locked control program would be implemented in the A/D interrupt subroutine. The interrupt program mainly includes the adjusting subroutine and the ADC interrupt subroutine (speed regulation subroutine, current adjusting subroutine). The mentioned above can be shown in the figure 4 and 5 . 


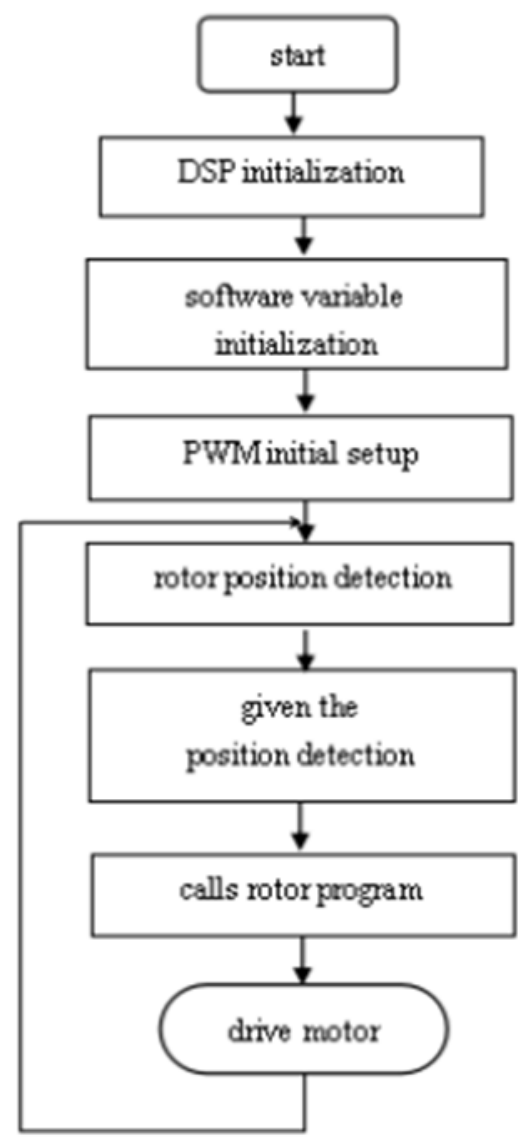

Figure 4. The main program flow chart

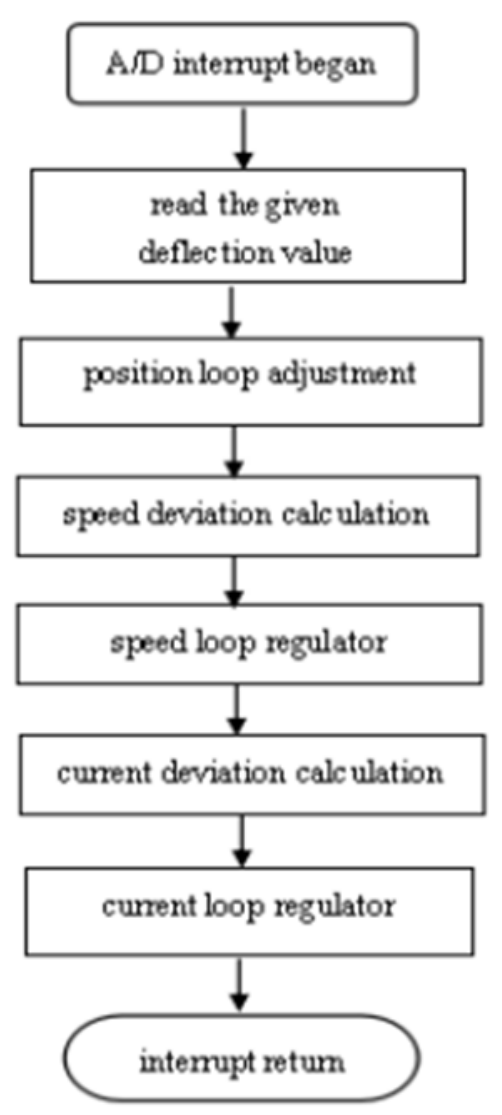

Figure 5. Subroutine flow chart

\section{The experimental results and conclusions}

As shown in the figures below, figure 6 and figure 7 are the simulation waveform of the motor when moving forwards. Figure 8 and figure 9 are the simulation waveform of the motor when moving backwards. The so-called VT is a counter, it is to be used to trigger signal. The PWM.CP represents the trigger state of PWM module. The pidl_spd.Fdb represents the feedback value of the speed and the speed is the speed of the motor. 

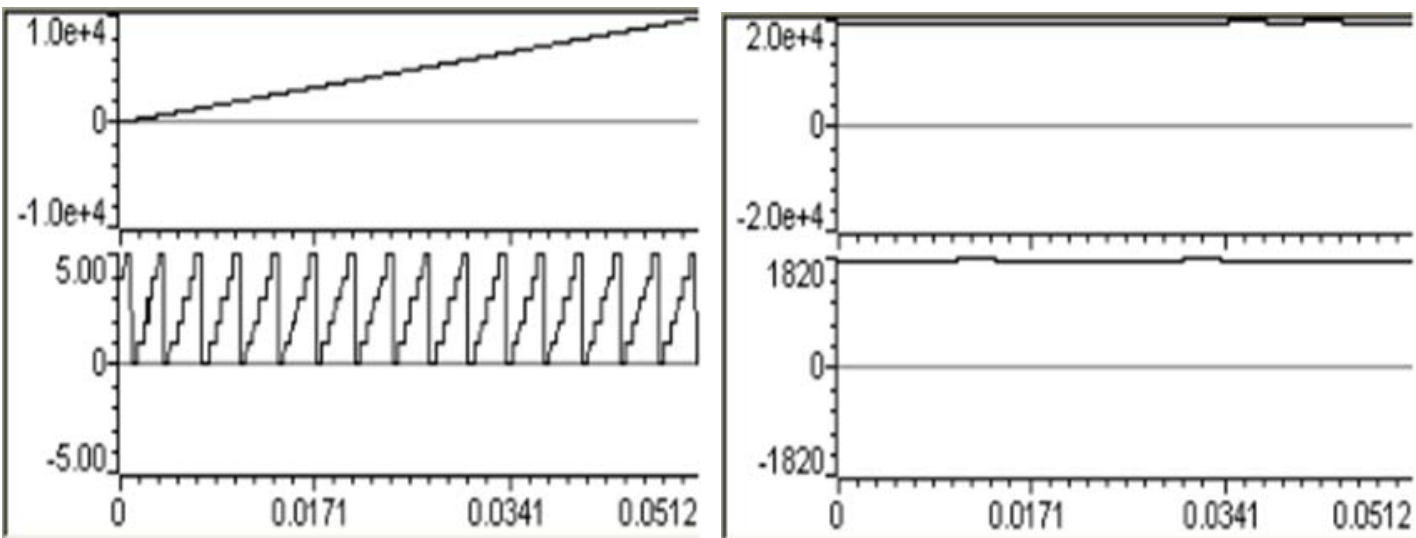

Figure 6. VT and PWM.CP waveform

Figure 7. pid1_spd.Fdb and speed waveform
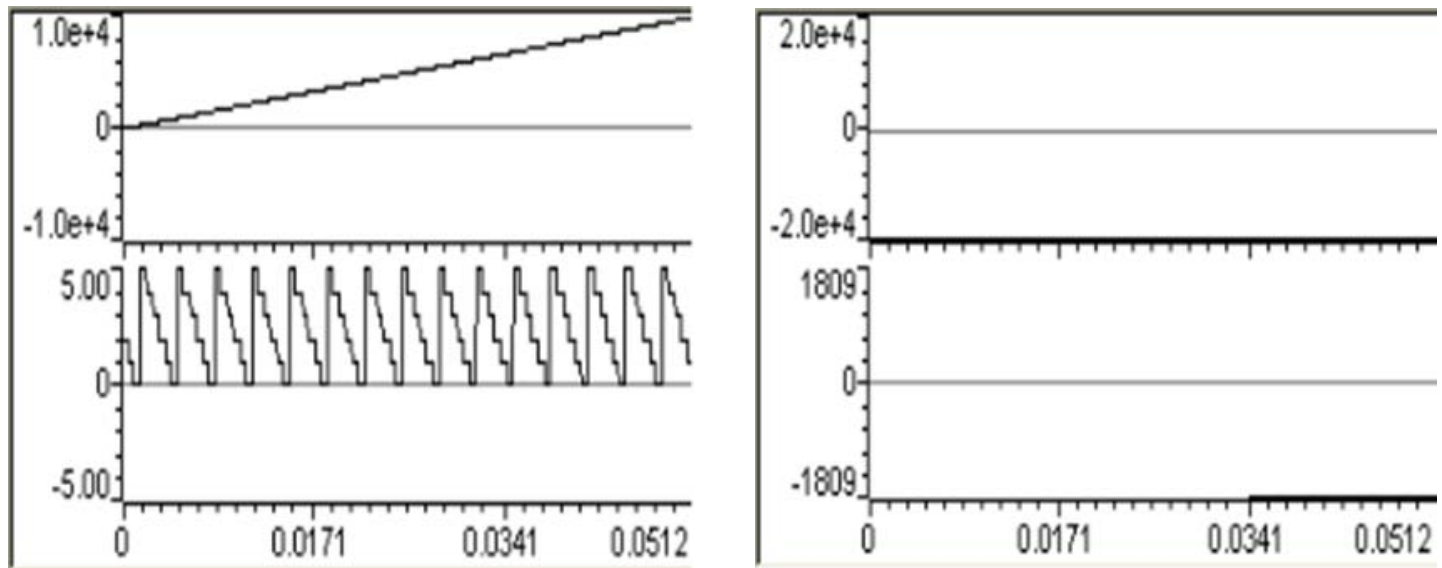

Figure 8. VT and PWM.CP waveform

Figure 9. Pid1_spd.Fdb and speed waveform

The paper proposed a design of double loop-locked control system of brush-less DC motor based on the TMS320F28335 DSP. It simplified the hardware structure of the traditional systems greatly by making full use of the rich on-chip resources and the efficient data processing ability of DSP. The design and implementation of control system of the brush-less DC motor adjust the actual system by adjusting the speed and the current closed-loops. The application of PID regulation of current loop and velocity loop makes the double loop-locked control system of brush-less DC motor to be small overshoot and fast response. After the analysis, we can conclude that the system has the advantage of low cost, stableand easy for implementation and extension, which makes great importance for engineering practice and research on motor's speed control.

\section{References}

1. J.P. Barrett, Electricity at the columbian exposition (Nadison: R R Donnelley, 1656)

2. M. Zhou, X.J. Fu, Trans. of CES, 30,103(2015)

3. N. Shinohara, S. Kawasaki, IEEE RWS, 13(2009)

4. J. Kurs, A. Karalis, Y. Moffatt, J. D. Joannopoulos, P. Fisher, M. Soljačić, Sci., 317, 83 (2007)

5. A. Karalis, J.D. Joannopoulos, M Soljačić, Ann. of Phys., 323, 34 (2008)

6. B. LUO, M.T. SHENG, S.C. XU, M. ZHANG, Proc. CSEE, 33, 171(2013)

7. L.Q. LI, S.D. HUANG, M.S. YANG, X.F. YUAN, Trans. CES, 28, 35(2013) 